\title{
Advanced Human-Computer Interface and Voice Processing Applications in Space
}

\author{
Julie Payette \\ Canadian Space Agency \\ Canadian Astronaut Program \\ St-Hubert, Quebec \\ J3Y 8 Y9
}

\begin{abstract}
Much irterest already exists in the electronics research community for developing and integrating speech technology to a variety of applications, ranging from voice-activated systems to automatic telephone transactions. This interest is particularly true in the field of aerospace where the training and operational demands on the crew have significantly increased with the proliferation of technology. Indeed, with advances in vehicule and robot automation, the role of the human operator has evolved from that of pilot/driver and manual controller to supervisor and decision maker. Lately, some effort has been expended to implement alternative modes of system control, but automatic speech recognition (ASR) and human-computer interaction $(\mathrm{HCI})$ research have only recently extended to civilian aviation and space applications. The purpose of this paper is to present the particularities of operator-computer interaction in the unique conditions found in space. The potential for voice control applications inside spacecraft is outlined and methods of integrating spoken-language interfaces onto operational space systems are suggested.
\end{abstract}

\section{INTRODUCTION}

For more than three decades, space programs internationally have been synonymous with the frontier of technological developments. Since 1957, NASA alone has launched an impressive series of earthorbiting satellites, exploration missions and manned vehicules.

Mission complexity has increased tremendously as instrumentation and scientific objectives have become more sophisticated. Recent developments in robotics and machine intelligence have led to striking changes in the way systems are monitored, controlled, and operated[1]. In the past, individual subsystems were managed by operators in complete supervisory and directing mode. Now the decision speed and complexity of many aerospace systems call for a new approach based on advanced computer and software technology.

In this context, the importance of the human computer interface cannot be underestimated. Astronauts will come to depend on the system interface for all aspects of space life including the control of the onboard environment and life support system, the conduct of experiments, the communication among the crew and with the ground, and the execution of emergency procedures.

One of the technology sought to help solve the human interface challenge in space is voice processing. Though Automatic Speech Recognition (ASR) and other forms of advanced voice I/O techniques have only recently been experimented with simple avionic systems, much remains to be done to meet the ergonomic characteristics of the unique operational environment found in space.

This paper presents the particularities of operator-computer interac- tion in space. As examples, the current environment of the Space Shuttle System is described and projected requirements for the international Space Station are examined. The paper also outlines the potential for voice control applications inside spacecraft and suggest methods of integrating spoken-language interfaces onto operational space systems.

\section{THE WORKPLACE: SPACE}

Any space flight represents some degree of risk and working in space, as in aviation, comports some hazards. Suddenly, at any time during a mission, a situation may occur that will threaten the life of the astronauts or radically alter the flight plan. Thus, critical to the success of the mission and security of the crew is the complex process of interaction between astronauts and their spacecraft, not only in routine operation, but also in unforseen, unplanned, and life-threatening situations.

\subsection{Environmental Factors}

The environment outside spacecraft is unforgiving. With surface temperatures ranging from $-180 \mathrm{C}$ in darkness and $440 \mathrm{C}$ in sunlight, high radiation and no atmosphere, lower earth orbit is hostile to life. Yet, astronauts work in this environment, under high workload and high stress, sheltered inside protective vehicules or dressed in bulky spacesuits.

To limit the risks of space walks, the ability to perform physical actions remotely is crucial. Aboard the shuttle, remote action is performed using of the Remote Manipulator System (RMS) ${ }^{1}$. And more than any other task performed in space, telerobotics introduces higher demands on the relationship between operators and machines[2].

Microgravity is another important environmental factors which considerably influence the interface configuration of aerospace systems. Space travel not only generates significant levels of stress in the human organism, but transforms the entire operational conditions. For instance, to perform their work in space, "weightless" astronauts must hold themselves "down" one way or another. They use straps and foot restraints, or simply grasp hand holders to maintain their position.

All the above considerations impose restrictions and introduce severe design requirements as follows:

- Safety: security is a paramount consideration aboard any

\footnotetext{
${ }^{1}$ The Remote Manipulator System of the Shuttle is a Canadian-built teleoperated robot am that is used in a semi- autonomous mode during those space flights that require objects to be handled, captured, and released into space.
} 
spacecraft. Every procedure and piece of equipment undergoes thorough review before being rated flight eligible. For example, all critical shuttle controls, such as an emergency stop switch, are required to meet very stringent layout requirements. No floating object or particle may inadvertently activate or damage a sensitive system.

- Reliability/Accuracy/Redundancy: high tolerance to failure is a condition to safety. Operative systems in space must be at least two fault-tolerant, if not more in the case of critical systems such as flight controls or environmental control and life support systems (ECLSS). Where applicable, error correction mechanisms must be implemented.

- Accessibility: the crew's ability to execute tasks safely and efficiently is notably improved if controls are ergonomically placed, clearly marked, and readily available[3]. Indirect accessibility is also crucial, particularly where overriding of automated functions is required.

- Feedback: in difficult operational environments such as microgravity, precise system feedback becomes essential. Through visual, auditive and tactile means, feedback reinforces security procedures and lessen the monitoring workload, particularly for telerobotic tasks which must be performed with extreme caution.

On the shuttle, for example, robot arm operations are executed by two astronauts, one manipulating the robot and the other assisting with secondary functions, camera controls and status displays. Visual feedback, if not precisely obtained from camera views, is directly available from the four windows of the Shuttle's flight deck. On Space Station, direct visual feedback will only be available on rare occasions and thus other means of feedback will have to be developed and integrated with the $\mathrm{HCI}$ of the robotics control workstation to provide camera redundancy.

- Commonality: system configuration consistent in type and quality for crew operations enhance efficacy and lowers training demands. Operating Space Station with an international crew, in particular, will necessitate very high commonality of functions to ensure safety.

\subsection{Technology Proliferation}

Environmental constraints are only one of the many factors influencing the $\mathrm{HCI}$ problem in space. Technological diversification is another. Recent advances have considerably increased the processing and information handling capability of computer systems, thus bringing additional operative complexity that must be absorbed by operators. In aircraft and spacecraft, despite notable efforts to integrate systems more efficiently, there is so much information, so many sources, datatypes, categories, variations, possibilities, layouts, scales, etc. that crew members no longer operate their system globally. Instead, they receive specific training or pair up to accomplish their tasks.

The impact of technology proliferation is clearly seen on the Space Shuttle. As described in a NASA technical report, it is clear "that the Shuttle cockpit contains the most complicated assortment of D\&C (Displays and Controls) ever developed for an aerodynamic vehicule. For control, there are toggle, push button, thumbwheel, press-down and rotary switches; potientiometers; keyboards; circuit breakers; and hand controllers. Display devices include circular and vertical meters, tape meters, mechanical talkbacks, annunciators, flight control meters, digital readouts and CRTs. There are more than 2100 D\&C devices in the orbiter cockpit."[3]

With the number and types of redundant subsystems continually increasing, the use of dedicated control devices is rapidly growing into a large, complex system difficult to update and interact with. These conditions have prompted reconsideration of the direction taken in aerospace system design.

An obvious solution to the problem of the exploding cockpit and crew workload in a demanding environment is a greater level of automation of functions and the introduction of alternative interfaces.

\section{ADVANCED INTERFACES}

The computer and operational systems used in space function under either autonomous or human control. Much of the configuration complexity is kept as transparent as possible to the users, to allow them to concentrate on the purpose of the interaction, rather than system design details. The current design approach focuses on means of simplifying operations wherever possible and facilitating operatormachine communication.

The concept of a more integrated human-computer system is clearly pertinent in space application. Astronauts are functional components of space systems, not only as operators and controllers, but as contributors to the overall performance of the system. On Space Station, where the network of computers will control and monitor thousands of automated systems as well as provide an interface to the crew, the need for performance will be heightened, necessitating increased automation and expansion of the supervisory role of the crew members.

However, the decision to automate certain aspects of aerospace mission operations demands a careful consideration of the potential human-computer relationship. The decision to use a machine for a particular set of functions will depend on many factors such as availability, appropriateness, cost, compatibility with existing systems, and more importantly, safety and efficiency.

Since few, if any, external resources and development systems will be available on a permanent space platform such as Space Station, great selectivity and perspicacity must be exercised when designing and building the human computer interface. Hence, the interest in investigating new forms of interfaces and input/output devices, such as voice command and automatic speech recognition.

\section{AUTOMATIC SPEECH IN SPACE}

Automatic recognition and understanding of speech is one of the very promising application of advanced information technology. As the most natural communication means for humans, speech is often argued as being the ultimate medium for human-machine interaction. On the other hand, with its hesitations and complexity of intention, spoken language is often thought as being inadequate and unsafe for accurate control and time critical tasks[4]. Unconvinced of the reliability of speech processing as a control technology, pilots and astronauts have traditionally been reluctant to accept voice interfaces.

Yet within a domain-limited command vocabulary, voice control has already been identified as a likely choice for controlling multifunction systems, displays and control panels in a variety of environments. Requiring minimal training, information transfer via voice control offers the basis for more effective information processing, 
particularly in situations where speakers are already busy performing some other tasks.

\subsection{Benefits of Speech Technology}

Motivations for using ASR in space are numerous. Traditionally, space operations have been accomplished via hardware devices, dedicated system switches, keyboards and display interfaces. In such context, ASR is seen as a complement to existing controls that should be used in conjunction with other interaction devices already bounded in terms of previously defined needs and capabilities.

It is foreseeable that voice control and synthesis could be used as an added I/O channel to use the crew more efficiently during peak workload periods. In particular, ASR may serve to facilitate operations in such areas as simultaneous control and monitoring (when hands and eyes are busy), extravehicular activities (EVA) and information storage and retrieval.

For some applications, voice commands combined with manual controls may allow more rapid task completion than would be possible with manual methods alone. As an example, a study conducted in the Manipulator Development Facility (MDF) of the NASA Johnson Space Center showed that voice control could be effectively used to perform the many switching camera functions associated with the closed-circuit television system supporting the RMS robot arm[3]. The study also revealed that identical tasks (berthing and deployment) were completed in virtually identical times using manual switching and voice controlled switching having recognition accuracy between 85 and 95 percent. Using more accurate, state-of-theart ASR equipment should allow for marked improvement in the overall RMS operations.

Interest in voice command and automatic speech recognition interfaces for space stems from the benefits it may bring to the demanding operational environment:

- hands free control

- altemate control (redundancy)

- extension capabilities

- task adaptability

- consistency of interface

- commonality of usage

- generic input/output function without requiring diversion of visual attention from monitoring tasks.

\subsection{Disadvantages and Concerns}

As described in section 2, technical constraints and environmental factors impose significant implementation requirements on the use of ASR and voice technology in space. Other issues to be considered range from the technical choices (isolated word vs continuous speech, single vs multiple speakers, word based vs phoneme based), the recognizer training update and maintenance requirements, the magnitude of changes in voice characteristics while in microgravity, and the effect of the space suit ( 0.3 atmosphere, pure oxygen) upon maintenance of highly accurate recognition.

Without a doubt, ASR system will require a very high recognition accuracy rate, possibly 99evaluations performed at NASA that astronauts will switch to habitual controls if latency, reliability and efficiency criteria are not met[5]. Also, safety and requirements will necessitate a high level of recognition feedback to the users, with interactive error correction and user query functions.

Finally, on the international Space Station, the diversity of languages and accents may make ASR an even more difficult challenge to meet.

\section{APPLYING VOICE IN SPACE}

Interest in voice technology for space applications is not new. NASA is actively pursuing applications of voice recognition and synthesis for its spacecraft and ground operations $[5,6,7,8]$. Several testbeds have incorporated voice into their commanding scheme, but only a few experiments have been performed in operational environments. These experiments are summarized below, followed by an outline of future applications.

\subsection{A Bit of History}

\section{Ground Test}

On the Shuttle, most Extra-Vehicular Activities (EVA) are performed for a specific tasks and rehearsed many times before the mission. To aid in these operations, cuff-mounted checklists have served as a useful reminder of procedures to follow. The problem with cuff checklists is that the wrist is not always in the best position for reading and at least one hand is required to turn the pages. Furthermore, information is limited to $3.25 \times 4.5$ inch pages which require a restraint to keep in position. For the longer missions on Space Station where EVA tasks will be less predictable, cuff checklist will be inadequate.

In 1986 and 1988, a voice-activated/voice-synthesis system was developed in conjunction with a prototype space suit to provide an alternate information system. Equipped with a voice-controlled head-mounted display, the suit was evaluated on the ground in a series of neutral buoyancy tests[9].

The voice system was termed an improvement over the cuff checklist, allowing both hands on the job while moving through procedures, but astronauts commented that the system created a lot of disruptive "chatter" on the channel and interfered with communications.

Voice Recording Test

In 1990, direct digital recordings of an astronaut's voice were performed on the ground before a mission, in flight during the mission and on the ground upon return. A selected vocabulary was used and templates were made. After analysis, significant acoustic differences were noted. No conclusions were drawn, however, as to whether microgravity was the cause of these changes in voice production, since the discrepancy was mostly blamed on a substantial difference between recording environments.

\section{Shuttle Flight Test}

To date, only one experiment using voice recognition technology has ever been used aboard a spacecraft. The voice command system (VCS) experiment flew on board Space Shuttle Discovery STS-41 in October 1990 and allowed astronauts Bill Shepherd and Bruce Melnick to control the closed-circuit television (CCTV) cameras and monitors by voice inputs[10]. The voice command system had the capability to control the CCTV camera selection, and camera functions such as pan, tilt, focus, iris and zoom. The VCS paralleled the manual controls and provided both audible and visual feedback. The system was speaker dependent with templates of the voice of the two astronauts previously made on the ground. The recognizer 
had limited continuous recognition and syntactic capabilities.

The VCS intended to collect baseline data on the effect of microgravity on speech production and recognition. In addition, the experiment was meant to show the operational effectiveness of controlling a spacecraft subsystem using voice input. Analysis of the data showed little variation between the microgravity and ground-based templates of the astronauts voices. According to the investigators, astronauts were pleased with the tests and stated that voice control was a useful tool for performing secondary tasks on the Shuttle.

Recent evaluations of different modes of camera control performed at NASA and to which the author participated have shown however that non-hardware controls will only be considered as sufficiently safe to be used in space if reliability can be proven, redundancy possible and efficiency significantly optimized. Moreover, as mentioned, experience has shown that crew members readily revert to the primary control system to which they are used to if an alternative system is not sufficiently accurate.

\subsection{Application Potential}

Spoken language communication with the control and monitoring subsystems onboard the Shuttle or Space Station is a convenience that could be provided through automatic speech recognition applications. Although ASR could not be the primary means of controlling critical actions [7], it could be used to backup the primary controller and as an alternative I/O medium for the crew.

Speech could also be used to query the status of a particular subsystem or database. Reference manuals could be called up and paged through. ASR could also be used for "hands-free" maintenance reporting, allowing the crew to attend to more important work and spend less time generating written reports.

Other possible applications would be the use of speech to overcome reduced manual dexterity caused by astronauts having to wear bulky space suit and gloves during ascent and reentry. Voice interfaces could be used to allow a diversity in the number of tasks to be performed as flexible as the size of the recognizer's vocabulary. Of particular interest are the high workload and adverse condition situations (G-load, noise, stress) where direct voice input could make a significant contribution to overall efficiency.

Another promising application for voice technology in space is during EVA activities, where voice control would allow astronauts on space walks to perform interactive queries and/or remote manipulator control, while busy performing some other maintenance or repair task or even simply, busy holding themselves down.

\subsection{Space Station}

The proposed Space Station may also benefit from some form of automatic voice interaction to reduce transaction time between crew members and their multitasking, multi-panel workstations. Spacebased crew are expected to interact with highly automated systems and to perform these interactions with often little prior training, or on an infrequent or sporadic basis. These activities will characterize a new role for astronauts, that of supervisory control.

For instance, current plans for the Space Station involve the use of a significant robotic workforce for assembly, servicing and maintenance tasks. Generically referred to as the Mobile Servicing System, this workforce will be operated from a multi- purpose control workstation. Equipped with three display devices, the workstation will include one keyboard, one cursor control device and a dedicated hardware switching panel. The HCI aspects of the workstation are currently under designed in Canada and the proposed configuration has already raised several major issues centering around how crewmembers will interact with multiscreen systems. As there will be times in which users will be performing up to four simultaneous tasks using the robotics workstation, designers are now looking at alternative methods of interaction, including voice-activated features.

Finally, as technology progresses, ASR might be used in conjunction with voice synthesis and natural language techniques to provide technical advice or even language translation to assist with communication between the international crew members.

\section{CONCLUSION}

Developing advanced human-computer interaction for space operations is a challenging task that requires the coordinated effort of various fields of study. A potential avenue for solution is to consider voice technology and automatic speech recognition techniques as means of optimizing astronaut performance and helping reduce their workload in space.

The voice experiment performed on the Space Shuttle mission STS41 in 1990 has demonstrated that an advanced voice system for interacting with on-board subsystems may prove both useful and cost effective.

Yet, as Dr. Vladimir Solov'yev, a veteran of two russian space missions totalling more than 12 months points out, the human component of the human-machine system remains a key:

Today, we have been accustomed to spacecraft launches and space flights are now perceived as something to be taken for granted. The experience of our cosmonauts is that there is no such thing as an easy space flight. A cosmonaut or ground control specialist is still a human being with its own set of capacities and problems. It means that it is a human being who takes a machine [up in space... and] a human, surrounded by a the hostile environment of space, who makes decisions and interacts with a machine to attain a desired result[11].

Any future HCI proposal will only achieve its purpose if it is designed with these limits in mind.

\section{References}

1. Heer and Lum editors. Machine Intelligence and Autonomy for Aerospace Systems. Progress in Astronautics and Aeronautics, volume 115, AIAA, 1988.

2. Smith T.J. and Stuart M.A. "Human Factors of Teleoperation in Space". In Proceedings of the Human Factors Society 34th Annual Meeting, 1990.

3. Burghduff, R.D. and Lewis, J.R. "Man-machine Interface and Control of the Shuttle Digital Flight System". In Proceedings of the Space Shuttle Technical Conference, Houston TX, 1983.

4. Howie, M.B., Woods, D.D. and Cook, R.I. "The Natural History of Introducing New Information Technology into a Highrisk Environment". In Proceedings of the Human Factors Society 34th Annual Meeting, 1990, pp. 429-433. 
5. Coler, C.R. "Use of Automatic Speech Recognition Technology for Space Applications". In Proceedings of the Voice IIO Systems Applications Conference, Palo Alto CA, 1984.

6. Parks, D.L. and White, R.W. Study to Determine Potential Flight Application and Human Factors Design Guidelines for Voice Recognition and Synthesis Systems. Boeing Commercial Airplane Company, NAS1-17367, 1985.

7. Sommers, M.J. "Capabilities and Constraints of Automatic Speech Recognition and its Use for the Space Station". In Proceedings of Speech Tech, New York, 1987, pp. 318-319.

8. Jordan, W.T. "Space Shuttle Voice Recognition System". In Proceedings of Speech Tech, New York, 1987, pp. 320-321.

9. Griffin, B.N. "Zero-G Simulation Verifies EVA Servicing of Space Station Modules". AIAA Space Station in the 21st Century, AIAA paper 86-2312, Reno NV, September 1986.

10. Salazar, G. "Voice Recognition Makes its Debut on the NASA STS-41 Mission". Speech Technology, Feb/March 1991, pp. 86-92.

11. Solov'yev, V. "Problems of Human-machine Interaction on Space Flight". In Humans and Machines in Space, Proceedings of the 29th Goddard Memorial Symposium, Johnson, May \& Kom editors, Univelt publishers, 1991, pp. 73-77. 